\title{
Thrombotic microangiopathy led to acute kidney injury in an infant with hemophagocytic lymphohistiocytosis: A case report
}

\author{
HAI-YAN GUO ${ }^{1}$, JINGMIN SUN ${ }^{1}$, YUE SU ${ }^{2}$, QIN ZHANG ${ }^{1}$, RAN HUA ${ }^{1}$, \\ $\mathrm{XUN}_{\mathrm{XIA}}{ }^{1}$ and DENG FANG ${ }^{3}$ \\ ${ }^{1}$ Department of Pediatrics, The First Affiliated Hospital of Anhui Medical University, Hefei, Anhui 230022, P.R. China; \\ ${ }^{2}$ Centre for Experimental Medicine, School of Medicine, Dentistry and Biomedical Sciences, \\ Queen's University Belfast, Belfast BT12 6BA, Northern Ireland, UK; \\ ${ }^{3}$ Department of Nephrology, Anhui Provincial Children's Hospital, Hefei, Anhui 230022, P.R. China
}

Received March 13, 2020; Accepted September 18, 2020

DOI: $10.3892 /$ etm.2021.9827

\begin{abstract}
Hemophagocytic lymphohistiocytosis (HLH) is a rare but fatal clinical syndrome frequently complicated by acute kidney injury (AKI) and acute tubular necrosis. Renal thrombotic microangiopathy (TMA) is a specific pathological feature of childhood HLH and few cases have been reported among infants. The present study presents a rare case of HLH with TMA in an infant. A 15-month-old infant with a week-long history of fever was admitted to hospital. The infant presented with AKI and subsequently a reduction in platelet and hemoglobin levels. TMA was diagnosed by kidney biopsy and the clinical, laboratory and bone marrow biopsy findings met the criteria of HLH. Due to a progressive increase in serum creatinine levels, hemodialysis was initiated on the second day following admission. Dexamethasone was administered to treat both the fever and HLH. The patient's body temperature returned to a normal range and platelet and hemoglobin levels were stable after 14 days of admission. Renal function stabilized on day 21 . The results of genetic testing did not identify any disease-related variations. Childhood HLH is a severe condition and mortality can be reduced by early diagnosis and correct treatment. For patients with HLH and AKI, the possible role of TMA should be considered. Renal biopsy can help to identify the cause of AKI and can be performed when the patient's condition is stable.
\end{abstract}

\section{Introduction}

Hemophagocytic lymphohistiocytosis (HLH), also known as hemophagocytic syndrome, is an abnormal immunoregulation syndrome characterized by excessive immune activation and

Correspondence to: Professor Deng Fang, Department of Nephrology, Anhui Provincial Children's Hospital, Hefei, Anhui 230022, P.R. China E-mail: dengfang@ahmu.edu.cn

Key words: hemophagocytic lymphohistiocytosis, thrombotic microangiopathy, acute kidney injury, infant hyperinflammatory disorder (1). HLH is generally divided into two types: Primary or familial HLH and secondary HLH (2). Primary or familial HLH results from genetic mutations, while secondary HLH occurs in infections, autoimmune diseases, malignancies, immunodeficiencies and organ transplantations $(2,3)$. The global incidence of HLH in children is 1-1.2 per 100,000 with a median age of onset of 1.8 years (4-7). Mortality in $\mathrm{HLH}$ ranges from $20-75 \%$ according to different reports, including children and adults $(3,6)$. After the International Histiocyte Society HLH-2004 treatment protocol was widely adopted, the 5-year survival rate of patients with this condition increased from 54 to $66 \%$ (8). According to these standards (2), identification of $\geq 5$ of the following indicators can be used to confirm HLH diagnosis: i) Fever; ii) splenomegaly; iii) cytopenia (affecting two or three lineages; hemoglobin $<90 \mathrm{~g} / \mathrm{l}$; platelets $<100 \times 10^{9} / 1$; neutrophil absolute value $\left.<1.0 \times 10^{9} / 1\right)$; iv) hypertriglyceridemia $(\geq 3 \mathrm{mmol} / \mathrm{l})$ and/or hypofibrinogenemia $(\leq 1.5 \mathrm{~g} / \mathrm{l})$; v) serum ferritin increase (>500 ug/l); vi) plasma soluble CD25 [soluble interleukin (IL)-2 receptor] increase $(\geq 2,400 \mathrm{U} / \mathrm{ml})$; vii) decrease or absence in natural killer (NK) cell activity; and viii) hemophagocytosis in the bone marrow, spleen or lymph nodes with no evidence of malignancy.

The onset of HLH is usually acute and the clinical manifestations of this critical illness are associated with pro-inflammatory cytokine storms and macrophage infiltration into tissue $(9,10)$. Cytotoxicity of impaired NK cells is the hallmark of HLH pathogenesis (11). Simultaneously, severe immune dysfunction contributes to excessive proliferation and persistent activation of antigen-presenting cells, uncontrolled cytokine secretion and proliferation, and ectopic migration of macrophages and T cells (12). Moreover, the high levels of pro-inflammatory cytokines, such as tumor necrosis factor (TNF)- $\alpha$, IL-6, and interferon (IFN)- $\gamma$, suppress hematopoiesis and lipoprotein lipase levels, resulting in cytopenia and hyperlipidemia $(9,10)$. These biological events are considered to be the causes of multiple organ failure and the high mortality of HLH (13).

Acute kidney injury (AKI) is a common complication of HLH. The global incidence of AKI concurrent with HLH varies from $8-62 \%$, with $33.3-59.0 \%$ of patients with 
AKI requiring renal replacement $(14,15)$. Thrombotic microangiopathy (TMA) is a rare disease characterized by microthrombus formation which obstructs the vascular cavity. Primary TMA can be induced by thrombotic thrombocytopenic purpura (TTP) and atypical hemolytic uremic syndrome (aHUS). Secondary TMA is often associated with the Shiga toxin, due to Escherichia coli, and pregnancy-related TMAs (16-18). However, TMA associated with AKI in combination with HLH is rarely reported. To the best of our knowledge, no reports have been published regarding infants, among whom this disease remains intractable (3). Due to the lack of characteristic clinical manifestations, providing a clear diagnosis of HLH remains challenging. The present study presents a case of HLH with TMA in an infant who showed a favorable prognosis after experiencing complications in diagnosis and treatment.

\section{Case report}

Patient information. A 15-month-old male presenting with a weeklong fever and reduced platelet and hemoglobin levels was admitted to Anhui Provincial Children's Hospital (Hefei, China) in November 2018. No improvement was observed after two days of antibiotic and antiviral treatment. No significant past medical history or family history was recorded.

Clinical findings. On admission, the axillary temperature of the patient was $41^{\circ} \mathrm{C}$, and the duration of the fever was prolonged. The patient's parents stated that the fever was accompanied by a chill and a facial rash, which subsided when the fever retreated. Physical examination revealed pharyngeal congestion and paleness.

\section{Diagnostic assessment}

Hematology test. The main features of the hematology test on the first day after admission indicated pancytopenia (hemoglobin, $9.3 \mathrm{~g} / \mathrm{dl}$; platelet count, $79 \times 10^{9} / 1$; neutrophil count, $2.79 \times 10^{9} / 1$ ), severe inflammation (C-reactive protein, $50.91 \mathrm{mg} / \mathrm{l}$; procalcitonin, $12.16 \mathrm{ng} / \mathrm{l}$ ), AKI (serum creatinine, $188 \mu \mathrm{mol} / \mathrm{l}$ ) and proteinuria (2+). Whole blood cell count decreased progressively (hemoglobin, $7.5 \mathrm{~g} / \mathrm{dl}$; platelet count, 26×10 $/ 1$; neutrophil count, $1.1 \times 10^{9} / 1$ ).

Concurrently, the causes of persistent fever and AKI remained unclear with the following tests reporting negative results: Hemoculture, Widal reaction, rheumatoid factor, antinuclear antibody series, antineutrophil cytoplasmic antibody, hepatitis A, hepatitis B, hepatitis $\mathrm{C}$ virus, syphilis, HIV, Legionella immunoglobulin M (IgM), mycoplasma IgM, chlamydia $\operatorname{IgM}$, rickettsia $\operatorname{IgM}$, adenovirus $\operatorname{IgM}$, respiratory and cytotoxic virus IgM, influenza A/B virus IgM and parainfluenza virus $\operatorname{IgM}$. The patient tested positive for the $\operatorname{IgG}$ antibodies of Epstein-Barr virus (EBV), cytomegalovirus (CMV) and the rubella virus, but the $\operatorname{IgM}$ antibody results were negative. In addition, tests for the presence of nucleic acids of EBV and CMV were negative. These findings suggested that the patient had previously contracted EBV, CMA and the rubella virus, but that these viruses were not active. Complement $\mathrm{C} 3$ and $\mathrm{C} 4$ were in the normal range (based on the observation in our hospital (The First Affiliated Hospital of Anhui Medical University, Hefei, China), C3 0.87-1.41 g/1, C4 0.1-0.4 g/l).
A blood smear did not detect any malarial parasites and the percentage of broken red blood cells was $1.3 \%$ (detailed information is described in Table SI).

Imaging examination. Low dose chest CT produced normal results and echocardiography did not show coronary artery dilation. At the acute phase, abdominal ultrasound suggested a diffuse enlargement of both kidneys (right kidney 104x33 mm, left kidney 100x32 mm), while the size of the liver and spleen were normal (data not shown).

Genetic testing. The strategy of genetic testing was whole-exome sequencing (WES) and the report showed there were two lysosomal-trafficking regulator (LYST) variants. The sequencing was performed by Beijing Chigene Translational Medicine Research Center Co., Ltd. The protocol of WES was as follows: i) Sample collection. The EDTA-treated peripheral blood was collected with informed consent of the patient's parents; ii) DNA extraction. The fetal tissue genomic DNA was extracted using a Blood Genome Column Medium Extraction kit (CW2298; CoWin Biosciences, Inc.) according to the manufacturer's instructions. The extracted DNA samples were subjected to quality control using a Qubit 2.0 fluorimeter and electrophoresis with $0.8 \%$ agarose gel; iii) whole exome library construction. Protein-coding exome enrichment was performed using xGen Exome Research Panel v1.0 (Integrated DNA Technologies, Inc.) that consisted of 429,826 individually synthesized and quality-controlled probes, which targeted a $39 \mathrm{Mb}$ protein-coding region (19,396 genes) of the human genome and covered $51 \mathrm{Mb}$ of end-to-end tiled probe space; and iv) sequencing. High-throughput sequencing was performed using an Illumina NovaSeq 6000 series sequencer (PE150; Illumina, Inc.) and $\geq 99 \%$ of the target sequence was sequenced. The next step was bioinformatics analysis: i) Quality control. Raw data were processed by fastp for adapter removal and low-quality read filtering; ii) Variant calling. The paired-end reads were performed using Burrows-Wheeler Aligner (BWA, version 0.7.11) to the Ensembl GRCh37/hg19 reference genome. Base quality score recalibration together with SNP and short indel calling was conducted using the Genome Analysis Toolkit. According to the sequence depth and variant quality, SNPs and indels were screened to ensure that high quality and reliable variants were obtained; iii) Variant annotation and pathogenicity prediction. The online system independently developed by Chigene (www.chigene.org; 12/21/2018) was used to annotate both database-based minor allele frequencies (MAFs) and the American College of Medical Genetics and Genomics practice guideline-based pathogenicity of every yielded gene variant (19). The system also provided serial software packages for conservative analysis and protein product structure prediction.

Therapeutic intervention. Initially, ceftriaxone and ganciclovir were administered due to the persistent fever. On the second day after admission, AKI was diagnosed by hematological parameters according to the Kidney Disease Improving Global Outcomes (KDIGO) AKI criteria of 2012 (20) of an increase in serum creatinine (Scr) within $48 \mathrm{~h}>26.5 \mu \mathrm{mol} / \mathrm{l}$ or Scr within 7 days up to 1.5 times that of the base value. Indicators for infection, such as C-reactive protein and procalcitonin levels, 
increased progressively. Therefore, hemodialysis was initiated and antibiotics were upgraded to meropenem and teicoplanin. Human serum albumin, intravenous immunoglobulin (IVIG), plasma and suspended red blood cell transfusion were administered to stabilize hemodynamics and low-dose dexamethasone (0.1-0.3 $\mathrm{mg} / \mathrm{kg} /$ day) was used as an anti-inflammatory and for suppression of the immune response.

Follow-up and outcomes. Serum ferritin levels increased progressively, from 950 to $2,237 \mu \mathrm{g} / 1$ within 6 days and serum IL-2 receptor levels were $>7,500 \mathrm{U} / \mathrm{ml}$ (normal range based on the observation in our hospital (The First Affiliated Hospital of Anhui Medical University, Hefei, China), 223-710 U/ml). The infant's fibrinogen levels sharply decreased from $4.27 \mathrm{~g} / 1$ on the third day of admission to $1.66 \mathrm{~g} / 1$ on the 26 th day (normal range based on the observation in our hospital (The First Affiliated Hospital of Anhui Medical University, Hefei, China), 2-4 g/l). Triglyceridemia increased progressively, from 1.75 to $5.07 \mathrm{mmol} / \mathrm{l}$. Moreover, NK cell count was initially normal, but decreased in the convalescent phase. Concurrently, the number of reticulocytes initially appeared to reduce but increased during the recovery period. Urine protein was between $2+$ and $3+$ throughout the period.

Imaging examination. During the recovery period, an abdominal ultrasound before discharge showed that the kidneys were in a recovery period from diffuse renal disease (right kidney 90x32 mm, left kidney 82x33 mm; data not shown).

To establish the pathogenesis of the fever, a bone marrow biopsy was performed on the sixth day of admission. Informed consent for this procedure was obtained from the patient's parents. A syringe was used to apply suction to the needle until marrow appeared. Tissue preparation: i) Prepare a film: A drop of bone marrow was placed onto one end of the smear slide. The spreader slide was placed at an angle of approximately $30-40^{\circ}$ in front of the drop and slid backwards until it came into contact with the sample drop. The spreader slide was advanced forwards, generating a smear with a feathered edge. ii) Smear staining: The smear was first dipped into methanol to fix the specimens and subsequently placed in Wright's-Giemsa stain (Beijing Solarbio Science \& Technology Co., Ltd.) for $10-15$ min for staining at room temperature $\left(20-25^{\circ} \mathrm{C}\right)$. The smear was next moved to a mixture of Wright's-Giemsa stain and $\mathrm{pH} 6.8$ phosphate buffer for $5 \mathrm{~min}$ at room temperature $\left(20-25^{\circ} \mathrm{C}\right)$. After the staining step, the smear was given a quick rinse in distilled water and was allowed to dry before cover-slipping. iii) Microscopy detection: Olympus BX53 (Olympus Corporation), Magnification, x100. HLH was considered due to the presence of mononuclear and phagocytic tissue cells in the bone marrow (Fig. 1). However, the recovery of renal function was not obvious after hemodialysis. To further clarify the etiology, a renal biopsy was performed on the 11th day of admission. Kidney tissue Preparation: Tissue for light microscopy (LM) was needed to keep the specimen in the vial of $10 \%$ formalin (Sigma-Aldrich; Merck KGaA) for 12-24 h. Regarding electron microscopy, the cortex with glomeruli (cut 1-2 mm from each end of the core) was placed in the vial that contained $2.5 \%$ glutaraldehyde solution (Sigma-Aldrich; Merck KGaA). Kidney tissue sectioning: The specimen was processed through dehydration, clearing, paraffin infiltration,

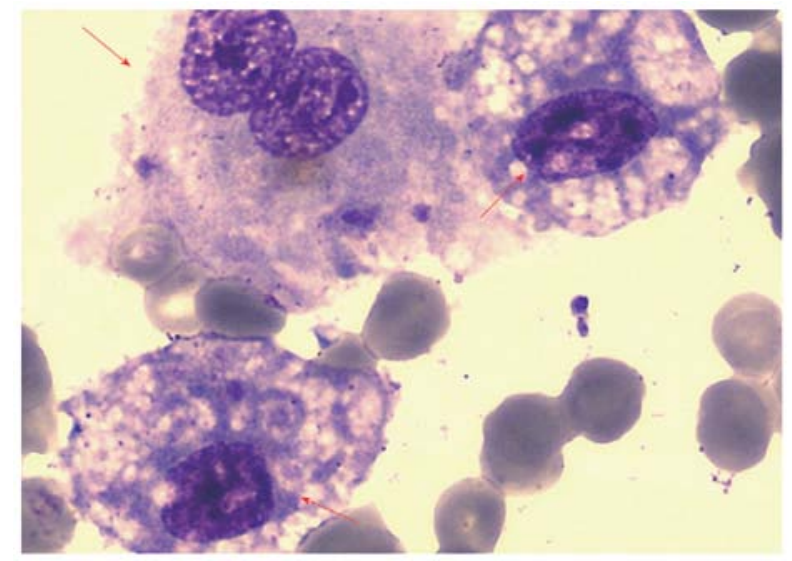

Figure 1. Bone marrow biopsy findings. Microscopic findings from bone marrow aspiration indicated phagocytosis of neutrophils by macrophages. Wright-Giemsa stain. Magnification, x100.

embedding and then sectioning the paraffin blocks at a thickness of 2-3 $\mu \mathrm{m}$. Kidney tissue staining: For light microscopy: Hematoxylin and eosin (H\&E) staining kit (Beyotime Institute of Biotechnology) and Masson stain kit (Abcam) were used for the staining. Image examination: Light microscope $\mathrm{x} 400$ (Olympus Corporation; BX53), scanning electron microscope $x 700$ (Hitachi, Ltd.). The glomeruli presented with diffuse minor lesions, a high number of microthrombus formations (5/11) and moderate tubulointerstitial lesions (Fig. 2). At this point, the child met the HLH diagnostic criteria and was consequently diagnosed with HLH complicated with TMA kidney damage.

Following HLH diagnosis, dexamethasone was administered according to the 2004 diagnostic and therapeutic guidelines for HLH (2). Due to the patient's age, immunosuppressive agents were not used. On the 14th day after admission, the patient's body temperature returned to normal and hemoglobin and platelet parameters were stable. On the 21st day of admission, serum creatinine levels was gradually decreased and the dialysis catheter was removed after six cycles of hemodialysis. The results of the auxiliary examination and treatment are shown in Fig. 3.

The cause of HLH in the patient remained unclear. Thus, genetic testing was performed. Within the whole-exome sequencing detection range, there was a variation in the LYST gene. However, no disease-related mutations were detected (Fig. 4). A follow-up examination after 18 months showed no sign of HLH recurrence and that serum creatinine levels were in the normal range.

\section{Discussion}

In the present case report, an infant was admitted to Anhui Provincial Children's Hospital with a persistent fever for 7 days. Laboratory tests showed anemia, thrombocytopenia and AKI. Initially, sepsis combined with multiple organ dysfunction was diagnosed, but detailed examinations did not support the diagnosis. Following renal biopsy and bone marrow aspiration, the diagnosis was clearly shown to be HLH. Although the clinical presentations of the patient were atypical at first, the infant met six indicators of the HLH-2004 

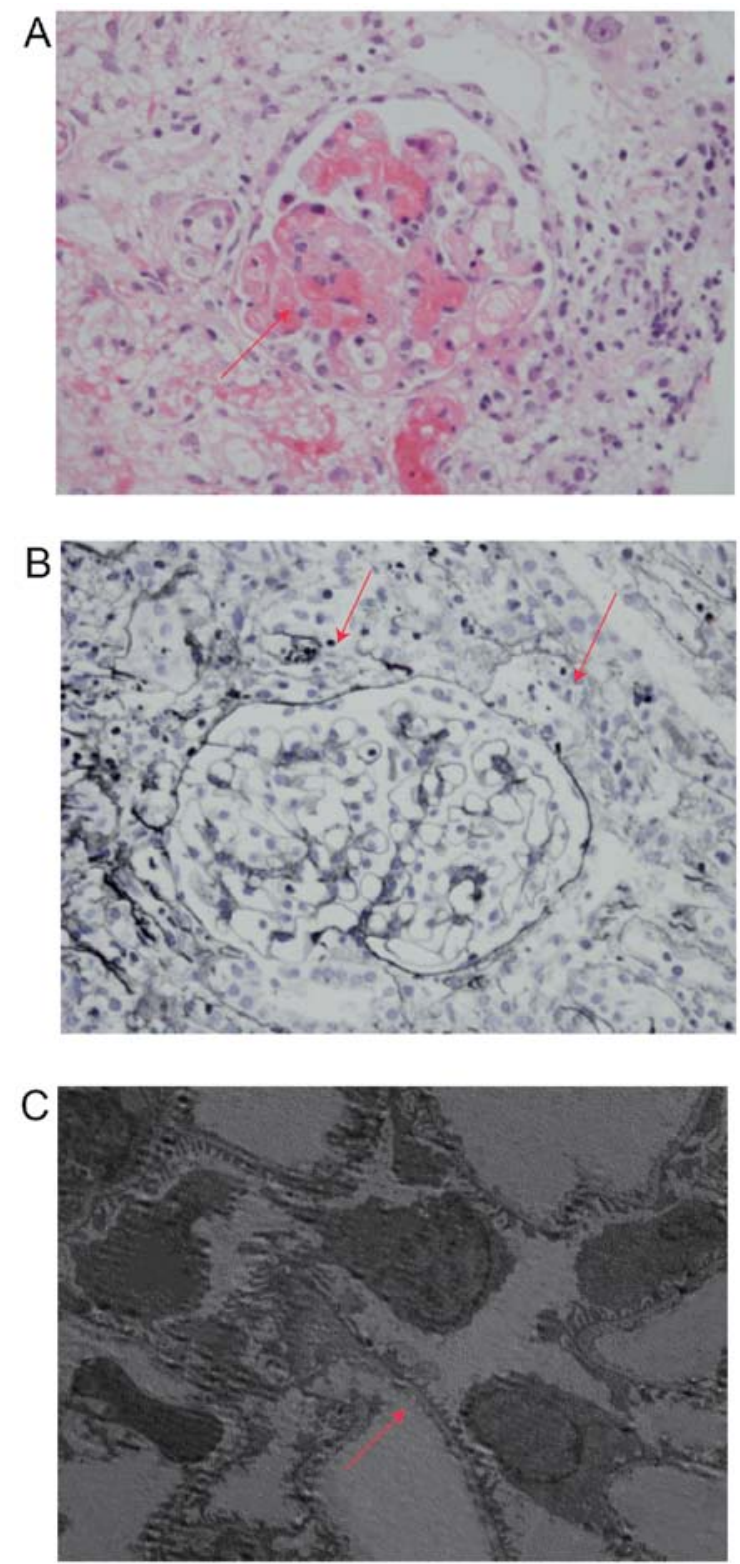

Figure 2. Kidney biopsy findings. (A) Light microscopic findings show capillary lumens filled with fragmented red blood cells. Hematoxylin and eosin stain. Magnification, $\mathrm{x} 400$. (B) Light microscopic findings demonstrate tubules focal moderate atrophy and renal tubular epithelial vacuoles and particle denaturation (Masson stain; magnification, $\mathrm{x} 400$ ). (C) Electron microscopy (magnification, $x 700$ ) findings demonstrated that the density of the segment inner tectorium of the glomerular basement membrane became thinner.

diagnostic criteria (2) on the 12th day after admission. Patients may not have all these clinical features on initial presentation and features may be accompanied by other symptoms, making diagnosis challenging (3).

In the present case, the initial increase in fibrinogen may be associated with the hypercoagulable state of the child, and fibrinogen levels decreased in the recovery stage due to the hypercoagulable improvement. Moreover, the reduction of reticulocytes at the acute phase and the rise during the recovery period may be associated with myelosuppression. In the present case, no spleen enlargement was noted during the course of treatment, which differed from the majority of HLH cases (2).

In the present case, rheumatism and tumor were excluded through the results of the examinations. Echocardiography did not show coronary artery dilation, and the patient did not present with Kawasaki-like symptoms. Moreover, although ganciclovir can induce AKI (21), the patient presented with renal dysfunction prior to ganciclovir treatment. AKI caused by prerenal and postrenal factors was excluded, as the patient did not show any signs of volume depletion and urinary tract obstruction. Atypical hemolytic uremic syndrome (aHUS) has many similar clinical features with HLH accompanied with AKI and TMA. According to the latest classification criteria presented at the KDIGO Controversies Conference in 2016 (22), $>50 \%$ of patients with aHUS have an underlying inherited and/or acquired complement abnormality, which results in dysregulated activity of the alternative pathway at the endothelial cell surface. In the present case, aHUS was not considered to be the diagnosis, since the complement $\mathrm{C} 3$ and $\mathrm{C} 4$ of the patient were in the normal range, the number of reticulocytes were reduced and the broken red blood cells in the peripheral blood were normal. The prognosis for aHUS is poor, as the disease will advance to end-stage renal disease within 2 years of presentation in the majority of patients who do not receive effective treatment such as eculizumab (22). During the 18-month follow-up, the renal function of the patient was normal, which indicated that the diagnosis of HLH with AKI and TMA was correct.

The accepted treatment protocol for HLH is based on 2004 guidelines (2), in which dexamethasone is recommended as the main therapy, while etoposide (VP16), methotrexate and cyclosporine A are major components of the treatment regimen (23). Additionally, hematopoietic stem cell transplantation (HSCT) is recognized as a recovery treatment (24). The main treatments administered in the present case report were dexamethasone and specific supporting therapy. Immunosuppressive agents were not used due to the patient's age. Supporting therapy, including dialysis and IVIG were administered and the clinical signs of the infant progressively improved. The present treatment protocol was in line with previous reports (25-28), in which immunosuppressive agents were not used and the outcome was also satisfying. Haytoglu et al (29) reported that patients with secondary HLH were treated with corticosteroids, IVIG and physical examination without chemotherapeutics and that hospital survival rate was $100 \%$. Therefore, the present case report presents evidence for the potential benefit of these therapeutic protocols, in which glucocorticoids (dexamethasone in this case) is the first-line drug and IVIG is recommended to all patients with HLH. As for the use of immunosuppressive agents, all clinical conditions should be taken into account and integrated and individualized treatments are hypothesized to be beneficial to patients.

In order to search the existing clinical cases of HLH with TMA, 'hemophagocytic lymphohistiocytosis' and 'renal thrombotic microangiopathy' were used as key terms when searching the PubMed database (https://pubmed.ncbi.nlm.nih.gov/). Clinical features of HLH with TMA in previously reported cases are summarized in Table I. Aside from the present case report, only one other case involving a child was reported, and the male to female ratio was $1: 1$. One case was infected with CMV, one case was infected with EBV, two cases were organ transplant patients, one was infected with parvovirus B19 and four cases were idiopathic. AKI was present in $7 / 8$ cases. Proteinuria was 

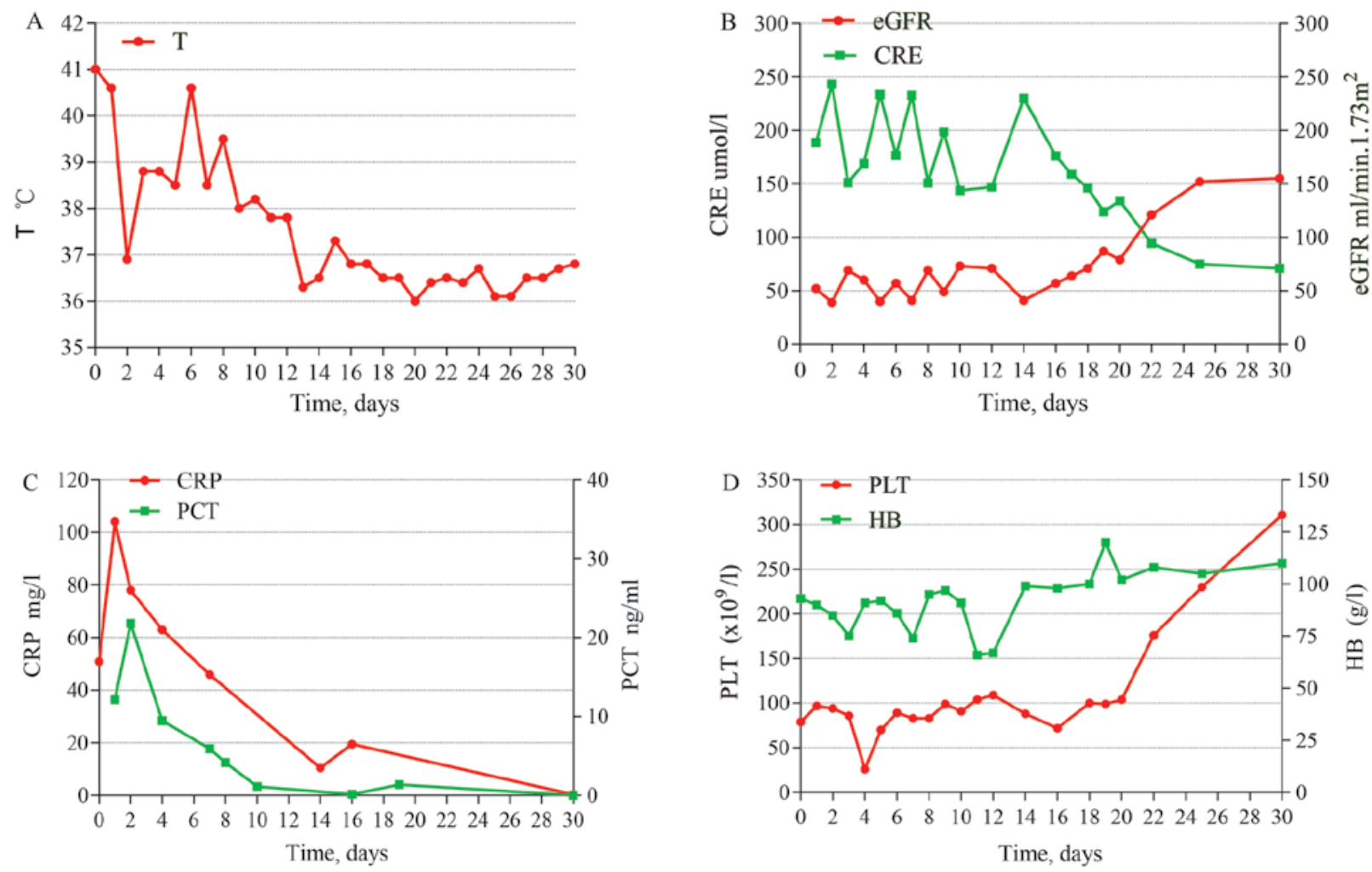

Figure 3. Body temperature and laboratory findings of the patient after admission. (A) A line graph indicating that the body temperature of the infant returned to normal at the 13th days after admission. (B) A renal function line graph demonstrates that CRE and eGFR stabilized to a normal range on the 21st day after admission. (C) An index of infection line graph indicated that the antibiotics were effective. The patient received antibiotics on the first day of admission. (D) Blood routine tests show that HB and PLT were stable at 22 days after admission. T, body temperature; eGFR, estimated glomerular filtration rate; CRE, serum creatinine; CRP, C-reactive protein; PCT, procalcitonin; HB, hemoglobin; PLT, platelet.

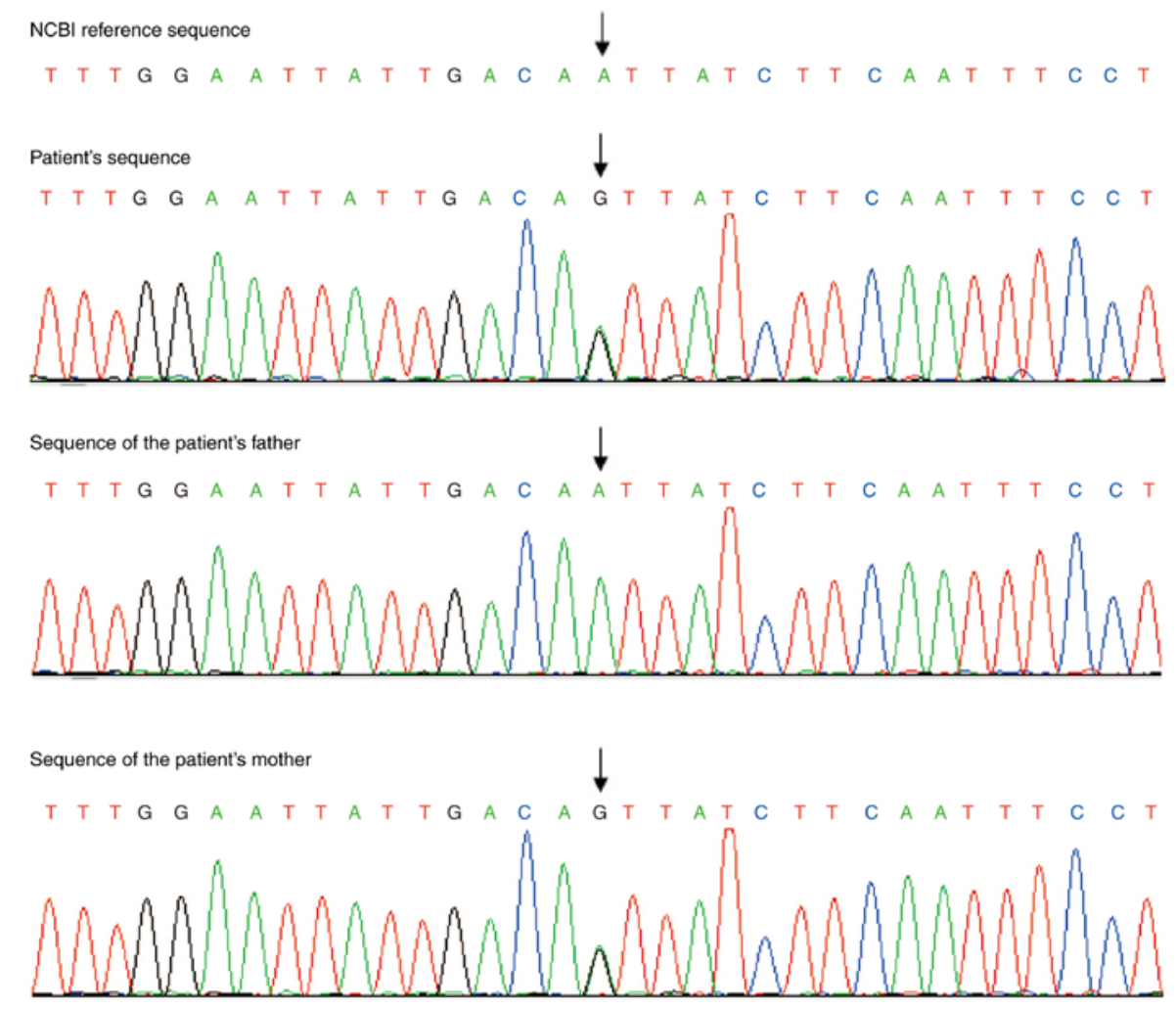

Figure 4. Sequence trace of WES in genetic testing. The first chromosomal location of LYST variant of this patient is in Chr1:235929469, the nucleotide change is c.6031 (exon21) A $>\mathrm{G}$ and the protein change is p. I2011V (p.Ile2011Val) (NM_000081). The second chromosomal location of LYST variant is in Chr1:235972624, the nucleotide change is c.1494 (exon5) A>G and the protein change is p. R498R (p.Arg498 Arg; NM_000081) LYST. LYST, lysosomal-trafficking regulator; WES, whole exome sequencing. 


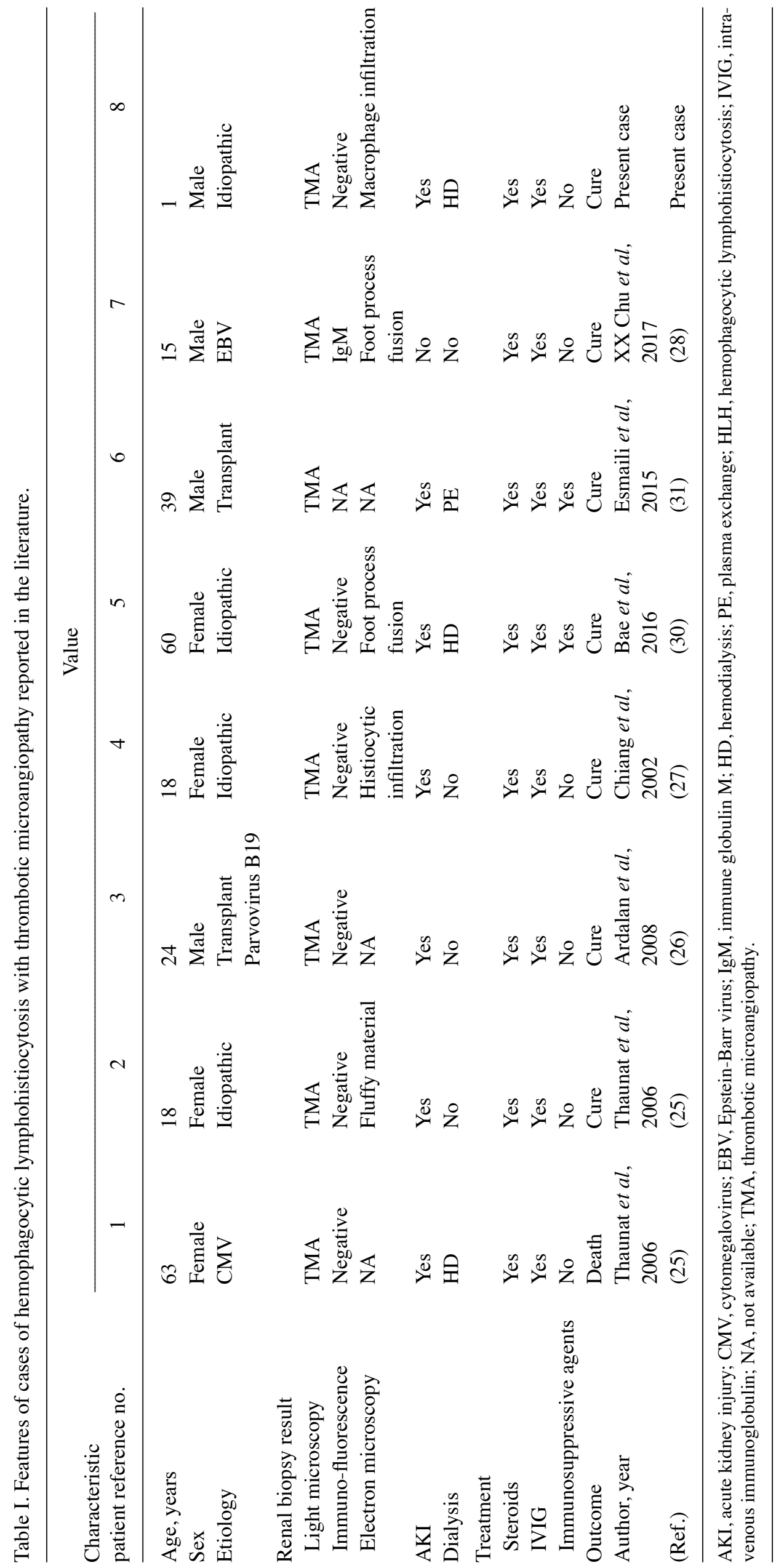


present in 5/8 cases. Dialysis was required for three patients and plasma exchange was required for one patient. Glucocorticoids and IVIG were administered in all patients. Immunosuppressive agents were only applied in two patients. Seven patients recovered and one patient succumbed to the disease.

One Japanese study reported that Mucor infection was associated with TMA in HLH (32). There were three cases of clinically-diagnosed HLH complicated TMA which did not undergo biopsy $(33,34)$. Bae et al (30) reported that TMA in HLH may occur with greater frequency than previously known, since diagnostic biopsies have rarely been performed in patients with HLH. If AKI occurred in patients with HLH, it is recommended that clinicians should also observe whether TMA is present in the patients as well.

The present case report hypothesized that the cause of the AKI was renal TMA, as the renal biopsy showed red blood cell debris obstructing the glomerular capillary lumen, severe tubular focal atrophy and microthrombosis in the arterioles. TMA is a histopathological lesion of the arteriole and capillary thickening, including intraluminal platelet thrombosis and vessel obstruction (17). In TMA pathogenesis, endothelial injury is a key factor in the initiation of microemboli formation $(35,36)$. The mononuclear-macrophage system is activated under the action of cytokines, releasing free radicals and proteolytic enzymes, resulting in endothelial cell damage and inflammatory reactions, thereby triggering TMA $(30,33)$. Similarly, systemically-activated lymphocytes and macrophages and overproduction of highly elevated proinflammatory cytokines in the circulation such as IFN, TNF, IL-6 and macrophage-colony stimulating factor are pathogenetic characteristics of HLH $(6,37)$. The immune dysfunction in TMA and in HLH has some overlap in pathophysiology, and clinicians should be aware of TMA when patients experience AKI with HLH.

Infections, malignancy and autoimmune disorders are the main causes of the secondary HLH (13). EBV is the most reported infection $(3,38)$, and several studies have reported that infections of CMV, herpes simplex viruses 1 and 2, varicella-zoster virus, roseolovirus, Kaposi's sarcoma-associated herpesvirus and Parvovirus B19 have been detected in secondary HLH $(13,25,26,39)$. However, the present case report involved blood EB virus antibodies, nucleic acid detection and renal tissue EB virus antibody detection, all of which were negative. Moreover, the present case report also excluded EBV infection, malignancy and autoimmune disorders.

Primary HLH is generally combined with clear familial inheritance or genetic problems, where it most often presents in the first year of life as an autosomal recessive disorder due to homozygous mutations (40). The annual incidence of primary HLH in Sweden is $0.12-0.15$ per 100,000 children per year (41). There are different types of familial HLH, including familial HLH types 2-5, X-linked lymphoproliferative disorder type 1, Griscelli syndrome type 2, Chediak-Higashi syndrome, X-linked lymphoproliferative disorder type 2, mutation of the Nod-like receptor family, caspase recruitment domain-containing 4, lysinuric protein intolerance and the mutation of heme oxygenase 1 , whereby the associated genes were PRF1, UNC13D, STX11, STXBP2, SH2D1A, RAB27A, LYST, BIRC4, NLRC4, SLC7A7, HMOX1 (13). LYST gene mutation may lead to pigment abnormalities due to abnormal melanocyte granules (42). It is worth noting that there is a variation in the LYST gene in the present patient, which can cause Chediak-Higashi syndrome (13), but most variations in the LYST gene are harmless to primary HLH (13) and there was no evidence demonstrating the association between LYST and this disease. The association between the LYST gene and hemophagocytic syndrome is still ambiguous, so at present, the etiology of HLH in the patient is not clear. Further analyses have demonstrated that $\sim 15 \%$ of patients with adult HLH harbor mutations in familial HLH genes that may serve as predisposing alleles for the development of HLH, though still in response to typical triggers (6).

Few large multicenter research studies have addressed the prognosis for children with HLH worldwide. A previous study (6) found that the survival rate of patients with HLH was only $10 \%$ before immunochemotherapeutic treatment strategies were administered, and once the HLH-94 guidelines were applied, the five-year overall survival rate was $54 \%$. Nandhakumar et al (3) revealed that 77\% (35/45) of children with secondary HLH recovered completely; however, all 13 patients with primary HLH died, despite the therapies administered according to the HLH 2004 protocol. To date, primary HLH has lacked effective treatment, and HSCT is recognized as the only viable treatment option to physicians. The latest clinical trial demonstrated that emapalumab is an efficacious targeted therapy for patients $<18$ years with primary HLH (43).

The present case report suggests that clinicians should consider TMA in patients with HLH with AKI. The major limitation of the present case report is the unknown pathogenesis of the disease. Since the genetic testing was negative and the follow-up inquiry into the child revealed that he had recovered, the cause of HLH was suspected to be an infection. However, the specific pathogenic microorganism causing this was not found, therefore, the present case is likely to be one of idiopathic HLH.

\section{Acknowledgements}

The authors would like to thank Mr. Zhiming Zheng (Chigene Translational Medicine Research Center Co., Ltd., Beijing, China), who performed whole exome sequencing for the patient.

\section{Funding}

The present study was supported by grants from the Anhui Province Public Welfare Technology Application Research Project (CN; grant no. 1704f0804027) and the Excellent Young Talents Fund Program of Higher Education Institutions of Anhui Province (grant no. gxbjZD07).

\section{Availability of data and materials}

The datasets used and/or analyzed during the current study are available from the corresponding author on reasonable request.

\section{Authors' contributions}

HYG performed the kidney biopsy, analysed the clinical data, and wrote the manuscript. JMS performed the histological examination and stated the presented diagnosis. YS interpreted the patient data and revised the manuscript. QZ and 
XX performed the kidney biopsy, performed the histological examination, and interpreted the patient data. $\mathrm{RH}$ analyzed and interpreted the patient's clinical data and contributed to writing the manuscript. DF analyzed and interpreted the patient data and revised the final manuscript. All authors read and approved the final manuscript..

\section{Ethics approval and consent to participate}

All procedures performed in the study were in accordance with the Ministry of Health ethics review on biomedical research involving human subjects, The declaration of Helsinki and the Council for International Organizations of Medical Sciences International ethical guidelines for biomedical research. Written informed consent was obtained from the family of the patient described in this report.

\section{Patient consent for publication}

Not applicable.

\section{Competing interests}

The authors declare that they have no competing interests.

\section{References}

1. Skinner J, Yankey B and Shelton BK: Hemophagocytic lymphohistiocytosis. Adv Crit Care 30: 151-164, 2019.

2. Henter JI, Horne A, Aricó M, Egeler RM, Filipovich AH, Imashuku S, Ladisch S, McClain K, Webb D, Winiarski J and Janka G: HLH-2004: Diagnostic and therapeutic guidelines for hemophagocytic lymphohistiocytosis. Pediatr Blood Cancer 48: 124-131, 2007.

3. Nandhakumar D, Loganatha A, Sivasankaran M, Sivabalan S and Munirathnam D: Hemophagocytic lymphohistiocytosis in children. Indian J Pediatr 87: 526-531, 2020.

4. Erker C, Harker-Murray P and Talano JA: Usual and unusual manifestations of familial hemophagocytic lymphohistiocytosis and langerhans cell histiocytosis. Pediatr Clin North Am 64: 91-109, 2017.

5. Allyson Niece M, Zora R. Rogers M, Naveed Ahmad M, Anne-Marie Langevin M and McClain KL: Hemophagocytic lymphohistiocytosis in Texas: Observations on ethnicity and race. Pediatr Blood Cancer 54: 424-428, 2010.

6. Allen CE and McClain KL: Pathophysiology and epidemiology of hemophagocytic lymphohistiocytosis. Hematology Am Soc Hematol Educ Program 2015: 177-182, 2015.

7. Henter J, Elinder G, Söder O and Ost A: Incidence in Sweden and clinical features of familial hemophagocytic lymphohistiocytosis. Acta Paediatr Scand 80: 428-435, 1991.

8. Bergsten E, Horne AC, Aricó M, Astigarraga I, Egeler RM, Filipovich AH, Ishii E, Janka G, Ladisch S, Lehmberg K, et al: Confirmed efficacy of etoposide and dexamethasone in HLH treatment: Long-term results of the cooperative HLH-2004 study. Blood 130: 2728-2738, 2017.

9. Frimmel S, Hinz M, Schipper J, Bogdanow S, Mitzner S and Koball S: Cytokine adsorption is a promising tool in the therapy of hemophagocytic lymphohistiocytosis. Int J Artif Organs 42: 658-664, 2019.

10. Zinter MS and Hermiston ML: Calming the storm in HLH. Blood 134: 103-104, 2019.

11. Egeler RM, ShapiroR,Loechelt B and Filipovich A: Characteristic immune abnormalities in hemophagocytic lymphohistiocytosis. J Pediatr Hematol Oncol 18: 340-345, 1996.

12. Usmani GN, Woda BA and Newburger PE: Advances in understanding the pathogenesis of HLH. Br J Haematol 161: 609-622, 2013.

13. Al-Samkari H and Berliner N: Hemophagocytic lymphohistiocytosis. Annu Rev Pathol 13: 27-49, 2018.
14. Aulagnon F, Lapidus N, Canet E, Galicier L, Boutboul D, Peraldi MN, Reuter D, Bernard R, Schlemmer B, Azoulay E and Zafrani L: Acute kidney injury in adults with hemophagocytic lymphohistiocytosis. Am J Kidney Dis 65: 851-859, 2015.

15. Karapinar B, Yilmaz D, Balkan C, Akin M, Ay Y and Kvakli K: An unusual cause of multiple organ dysfunction syndrome in the pediatric intensive care unit: Hemophagocytic lymphohistiocytosis. Pediatr Crit Care Med 10: 285-290, 2009.

16. Bayer G, Von Tokarski F, Thoreau B, Bauvois A, Barbet C, Cloarec S, Mérieau E, Lachot S, Garot D, Bernard L, et al: Etiology and outcomes of thrombotic microangiopathies. Clin J Am Soc Nephrol 14: 557-566, 2019.

17. George JN and Nester CM: Syndromes of thrombotic microangiopathy. N Engl J Med 371: 654-666, 2014.

18. Barbour T, Johnson S, Cohney S and Hughes P: Thrombotic microangiopathy and associated renal disorders. Nephrol Dial Transplant 27: 2673-2685, 2012.

19. Richards S, Aziz N, Bale S, Bick D, Das S, Gastier-Foster J, Grody WW, Hegde M, Lyon E, Spector E, et al: Standards and guidelines for the interpretation of sequence variants: A joint consensus recommendation of the American College of Medical Genetics and Genomics and the Association for Molecular Pathology. Genet Med 17: 405-424, 2015.

20. Kidney Disease: Improving Global Outcomes: KDIGO Clinical Practice Huideline for Acute Kidney Injury. KDIGO AKI Guideline, Online Appendices A-F, 2012.

21. Dos Santos Mde F, Dos Santos OF, Boim MA, Razvickas CV, De Moura LA, Ajzen H and Schor N: Nephrotoxicity of acyclovir and ganciclovir in rats: Evaluation of glomerular hemodynamics. J Am Soc Nephrol 8: 361-367, 1997.

22. Goodship TH, Cook HT, Fakhouri F, Fervenza FC, Frémeaux-Bacchi V, Kavanagh D, Nester CM, Noris $M$, Pickering MC, Rodríguez de Córdoba S, et al: Atypical hemolytic uremic syndrome and C3 glomerulopathy: Conclusions from a 'Kidney Disease: Improving Global Outcomes' (KDIGO) controversies conference. Kidney Int 91: 539-551, 2017.

23. Sadaat M and Jang S: Hemophagocytic lymphohistiocytosis with immunotherapy: Brief review and case report. J Immunother Cancer 6: 49, 2018.

24. Schram AM and Berliner N: How I treat hemophagocytic lymphohistiocytosis in the adult patient. Blood 125: 2908-2914, 2015.

25. Thaunat O, Delahousse M, Fakhouri F, Martinez F, Stephan JL, Noël LH and Karras A: Nephrotic syndrome associated with hemophagocytic syndrome. Kidney Int 69: 1892-1898, 2006.

26. Ardalan MR, Shoja MM, Tubbs RS, Esmaili H and Keyvani H: Postrenal transplant hemophagocytic lymphohistiocytosis and thrombotic microangiopathy associated with parvovirus B19 infection. Am J Transplant 8: 1340-1344, 2008.

27. Chiang WC, Wu MS, Tsai CC, Lin SL, Tsai TJ and Hsieh BS: Thrombotic microangiopathy in hemophagocytic syndrome: A case report. J Formos Med Assoc 101: 362-367, 2002.

28. XX Chu, Zhang Y and Liao SJ: A case of HLH complicated with renal damage of thrombotic microangiopathy. Chin J Nephrol 33: 625-626, 2017.

29. Haytoglu Z, Yazici N and Erbay A: Secondary hemophagocytic lymphohistiocytosis: Do we really need chemotherapeutics for all patients? J Pediatr Hematol Oncol 39: e106-e109, 2017.

30. Bae MN, Kwak DH, Park SJ, Choi BS, Park CW, Choi YJ, Lee JW, Yang CW, Kim YS and Chung BH: Acute kidney injury induced by thrombotic microangiopathy in a patient with hemophagocytic lymphohistiocytosis. BMC Nephrol 17: 4, 2016.

31. Esmaili H, Mostafidi E, Mehramuz B, Ardalan M and Mohajel-Shoja M: An update on renal involvement in hemophagocytic syndrome (macrophage activation syndrome). J Nephropathol 5: 8-14, 2016.

32. Inagaki N, Sugimoto K, Hosone M, Isobe Y, Yamamoto Y, Sasaki M, Kato A, Mori T and Oshimi K: Disseminated Mucor infection and thrombotic microangiopathy in lymphoma-associated hemophagocytic syndrome. Int J Hematol 88: 355-356, 2008.

33. Ardalan MR: Review of thrombotic microangiopathy (TMA), and post-renal transplant TMA. Saudi J Kidney Dis Transpl 17: 235-244, 2006

34. Ishiguro T, Kojima A, Shimizu T, Mita N, Kuroiwa S and Takayanagi N: Combined hemophagocytic syndrome and thrombotic microangiopathy due to mixed infection with influenza virus and pneumococcal pneumonia. Clin Case Rep 7: 131-134, 2019. 
35. Yu XJ, Yu F, Song D, Wang SX, Song Y, Liu G and Zhao MH: Clinical and renal biopsy findings predicting outcome in renal thrombotic microangiopathy: A large cohort study from a single institute in China. ScientificWorldJournal 2014: 680502, 2014.

36. Goldberg RJ, Nakagawa T, Johnson RJ and Thurman JM: The role of endothelial cell injury in thrombotic microangiopathy. Am J Kidney Dis 56: 1168-1174, 2010.

37. Canna SW and Marsh RA: Pediatric hemophagocytic lymphohistiocytosis. Blood 135: 1332-1343, 2020.

38. Mărginean MO, Molnar E and Chinceşan MI: Epstein-Barr virus-associated hemophagocytic lymphohistiocytosis in a small child: A case report. Medicine (Baltimore) 99: e18759, 2020.

39. MunozJ, ShareefN and Donthireddy V: Cytomegalovirus-induced haemophagocytic lymphohistiocytosis syndrome. BMJ Case Rep 2012: bcr1020114963, 2012.

40. Esteban YM, de Jong JLO and Tesher MS: An overview of hemophagocytic lymphohistiocytosis. Pediatr Ann 46: e309-e313, 2017.
41. Meeths M, Horne A, Sabel M, Bryceson YT and Henter JI: Incidence and clinical presentation of primary hemophagocytic lymphohistiocytosis in Sweden. Pediatr Blood Cancer 62: 346-352, 2014

42. Risma KA and Marsh RA: Hemophagocytic lymphohistiocytosis: Clinical presentations and diagnosis. J Allergy Clin Immunol Pract 7: 824-832, 2019.

43. Locatelli F, Jordan MB, Allen C, Cesaro S, Rizzari C, Rao A, Degar B, Garrington TP, Sevilla J, Putti MC, et al: Emapalumab in children with primary hemophagocytic lymphohistiocytosis. N Engl J Med 382: 1811-1822, 2020.

This work is licensed under a Creative Commons Attribution-NonCommercial-NoDerivatives 4.0 International (CC BY-NC-ND 4.0) License. 